\title{
Carrageenan-Induced Non-Painful Inflammation Reduces Voluntary Sucrose Consumption in a Two-Bottle Choice Paradigm: A New Model of Inflammation Induced Anhedonia
}

\author{
Marco A. Sotomayor-Sobrino', Abraham Ochoa-Aguilar¹, Ulises Coffeen², \\ Roberto Velasco-González ${ }^{1}$ Gilberto Pérez-Sánchez², Gloria M. Capula-León², \\ Israel Luna-Mendoza1, Lenin Pavón-Romero², Claudia Gómez-Acevedo ${ }^{1 *}$ \\ ${ }^{1}$ Pharmacology Department, Faculty of Medicine, National University of Mexico, Mexico City, Mexico \\ ${ }^{2}$ Neuroscience Division, National Institute og Psychiatry “Ramón de la Fuente Muñiz", Mexico City, Mexico \\ Email: *goac@unam.mx
}

How to cite this paper: Sotomayor-Sobrino, M.A., Ochoa-Aguilar, A., Coffeen, U., Velasco-González, R., Pérez-Sánchez, G., Capula-León, G.M., Luna-Mendoza, I., Pavón-Romero, L. and Gómez-Acevedo, C. (2020) Carrageenan-Induced Non-Painful Inflammation Reduces Voluntary Sucrose Consumption in a Two-Bottle Choice Paradigm: A New Model of Inflammation Induced Anhedonia. Journal of Behavioral and Brain Science, 10, 153-164. https://doi.org/10.4236/jbbs.2020.103009

Received: January 17, 2020

Accepted: March 21, 2020

Published: March 24, 2020

Copyright () 2020 by author(s) and Scientific Research Publishing Inc. This work is licensed under the Creative Commons Attribution International License (CC BY 4.0).

http://creativecommons.org/licenses/by/4.0/

\begin{abstract}
Anhedonia, the presence of diminished pleasure or decreased motivation for rewards is considered one of the core symptoms of depression. Studies have shown that anhedonia and reduced reward learning predict a poor prognosis with a higher prevalence of treatment failure among patients. Several mechanisms have been implicated in the onset and progression of depression; furthermore, an important amount of evidence supports not only the importance of the reward circuitry in the development of depressive anhedonia but also, that it is closely related with the presence of inflammation. In the current paper we show that 500 ul carrageenan administration in the right hind paw after deafferentation surgery leads to anhedonia, which is evident by a decrease in saccharine consumption in a two-bottle choice test; these findings suggest that carrageenan mediated inflammation is a reliable model of anhedonia that could lead to a better understanding of the immune-neural axis in motivation.
\end{abstract}

\section{Keywords}

Anhedonia, Inflammation, Animal Model, Motivation

\section{Introduction}

Anhedonia, the presence of diminished pleasure or decreased motivation for rewards is considered one of the core symptoms of depression [1] [2]. Studies have 
shown that anhedonia and reduced reward learning predict a poor prognosis with a higher prevalence of treatment failure among patients [3] [4]. The number of depressive patients with anhedonia suggests that aberrant reward processing, due to changes in reward circuitry, is an important mechanism underlying depression pathophysiology [5].

Currently, several mechanisms have been implicated in the onset and progression of depression; including the HPA axis dysregulation, the presence of genetic receptor alterations and dopamine deficiencies in the mesolimbic axis [6] [7] [8]. Furthermore, an important amount of evidence supports not only the importance of the reward circuitry in the development of depressive anhedonia but also, that it is closely related with the presence of inflammation [9] [10]. Evidence has shown that pro-inflammatory cytokines, in addition to their effect in the periphery, can communicate with the brain and induce depressive-like behaviors, including anhedonia, decrease in exploratory, novelty-seeking and social behaviors, as well as reduce food intake and sleep disturbance [10] [11] [12] [13]. Experiments performed by Yirmiya [14] showed that endotoxin-(e.g. lipopolysaccharide) and cytokine-induced anhedonia in rodents is often described in terms of reduced intake of palatable substances (e.g. sweetened water or milk). Recently, this reduction in consumption of palatable substances has been observed in various animal procedures that induce depression, including chronic mild stress, olfactory bulbectomy and social stress [15] [16] [17].

Additionally, it has been reported that carrageenan-induced inflammation attenuates place preference produced by the administration of morphine, cocaine and methamphetamine; suggesting that the inflammation overshadows the drug stimulus and inhibits the place-preference conditioning otherwise showed by the administration of morphine, cocaine and methamphetamines [18]. However, carrageenan-induced inflammation has not been considered a model of anhedonia because of the associated pain observed. Therefore, in the present study, we investigated if the deafferentation of the sciatic nerve could eliminate the pain component in the carrageenan-induced inflammation, and if it would be suitable as an anhedonia model.

\section{Methods}

Experiments reported here were conducted in the Pharmacology Department at the Faculty of Medicine of the National University of México (UNAM) and in the Neuroscience Division of the National Institute of Psychiatry. In both cases, the protocol was approved by the Research and Ethics Committee of the Faculty of Medicine and that of the National Institute of Psychiatry, in compliance with the Animal's Protection Act and the General Health Law. Surgical procedures were carried out based on the guidelines of the Ethics Committee of the International Association for the study of pain [19].

\subsection{Animals}

Male Wistar rats between 250 and $350 \mathrm{~g}$ of weight, and 5 to 8 weeks of age, were 
housed in groups of 3 to 5 rats per cage. Animals had access to food and water Ad libitum and were subjected to an inverted $12 \mathrm{~h}$ light/dark cycle (lights on from 18:00 to 06:00 h). All efforts were made to minimize animal suffering and to use only the number of animals necessary to produce reliable scientific data.

All behavioral tests were performed in a room with dim red illumination and dampening external noise during the active phase of rat's diurnal cycle. Before the beginning of the experiments, all animals were handled daily by the experimenter for a week and on the day of the experimental sessions' animals were left in the experimental room for an hour in order to habituate to the surroundings.

\subsection{Surgical Technique}

As mentioned before, to eliminate the pain component of the carrageenan-induced inflammation, the deafferentation of the sciatic nerve was performed as described elsewhere [20] [21]. Briefly, after mild sedation with intraperitoneal pentobarbital $(30 \mathrm{mg} / \mathrm{kg})$, chlorhexidine was applied topically over the area of foray of the right hind leg. Then, $0.5 \mathrm{ml}$ of lidocaine $(20 \mathrm{mg} / \mathrm{ml})$ was administered in the area close to the pelvic articulation. Few minutes later, the sciatic nerve was exposed through an incision (10 $\mathrm{mm}$, approximately) over the pelvic joint. The muscle fibers were carefully separated to expose the sciatic nerve and $0.3 \mathrm{ml}$ of lidocaine $(20 \mathrm{mg} / \mathrm{ml})$ was topically applied at the nerve. The efficacy of the pain blockage was assessed using paw withdraw latency and vocalization in response to nociceptive stimulus using a clamp in the ipsilateral paw for 60 seconds after the topical administration of the anesthetic drug. Finally, a $2 \mathrm{~mm}$ segment of the nerve proximal to its bifurcation into the tibial and common peroneal nerves was removed, and the incision was sutured. Animals were allowed to recover from surgery in a dim light area and corporal temperature was maintained using an electrical thermic pad. Animals were closely monitored and those who showed signs of distress or pain were withdrawn from the protocol.

\subsection{Carrageenan-Induced Inflammation}

A volume-response curve was performed by injecting $500 \mathrm{ul}$ of $1 \%$ carrageenan (carrageenan type IV, Sigma-Aldrich) diluted in saline in the right hind foot pad. Paw volumes were determined using a water plethysmometer.

\subsection{Thermal Hypersensitivity and Mechanical Allodynia Tests}

Before pain response evaluation, animals were daily habituated for $15 \mathrm{~min}$ in the testing apparatus during three consecutive days. Baseline recordings were performed before the deafferentation of the sciatic nerve, and again a week after surgery. Additionally, right hind paw volumes were assessed and $1 \%$ carrageenan (500 ul) was administered in the right hind foot pad. Thermal hypersensitivity and mechanical allodynia tests were daily performed for 7 days.

Thermal hypersensitivity was evaluated using a plantar test apparatus, as previously reported [22]. Briefly, an infrared radiant heat source was focused on the middle of the plantar surface of the hind paw, and time taken for the paw with- 
drawal was measured automatically. To avoid thermal injury, the cutoff duration of exposure was set at $12 \mathrm{~s}$. The withdrawal latencies for three trials performed at 5 min interval were averaged.

Mechanical nociceptive thresholds were evaluated using an automated Von Frey-type dynamic plantar esthesiometer, as described before [22] [23]. Briefly, each rat was placed in a plastic cage with a wire mesh floor. The tip of a von Frey filament was applied to the middle of the plantar surface of the hind paw with increasing force until the paw was withdrawn or a cutoff force of $50 \mathrm{~g}$ was reached. Both, latency to withdraw and the force required to elicit this reflex withdrawal of the hind paw were automatically recorded. To avoid any injury, the cutoff duration of exposure was set at $10 \mathrm{~s}$. Data represent the average of four trails performed at 5 min interval.

\subsection{Two-Bottle Choice Saccharin Preference Test}

A two-bottle choice procedure was used to test saccharin preference (Figure 1). For this test, rats were housed individually; on the first day rats received two drinking bottles in their home cage filled with tap water to get used to the two bottles. The following day, one of the two drinking bottles was filled with a $0.1 \%$ saccharin solution (Sigma-Aldrich). Twenty-four hours later, the two bottles were weighed, refilled with water or saccharin solution and placed in reversed order. Twenty-four hours later, bottles were weighed again. Consumption was registered, before carrageenan administration, for three consecutive days and for 7 days after. Saccharin preference was expressed as average daily intake of saccharin solution to the total daily fluid intake (as a percentage).

\subsection{Prostaglandin Analysis}

Prostaglandin 2 (PGE2) plasma concentration was measured after administration of carrageenan. Blood was collected using retro-orbital bleeding technique described

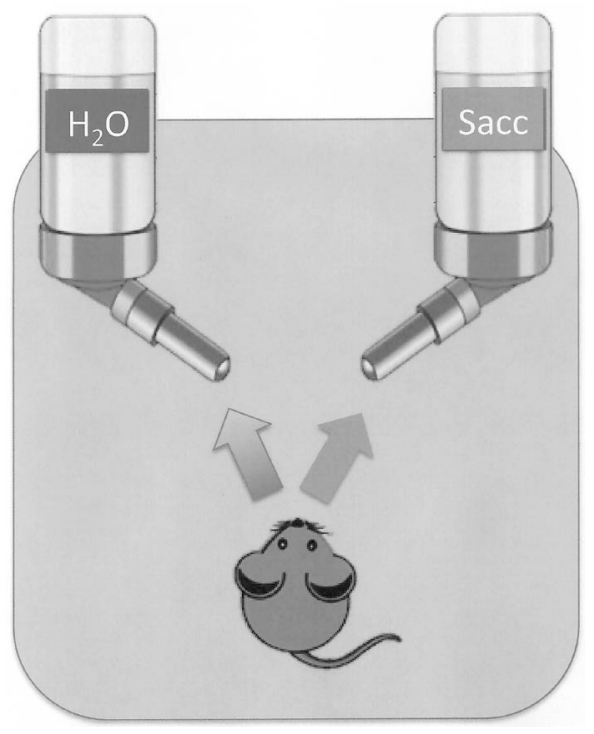

Figure 1. Two-bottle choice paradigm. 
elsewhere (Sharma, A., et al., 2014). Briefly, animals were anesthetized with pentobarbital $(30 \mathrm{mg} / \mathrm{kg}$, i.p.), and a sample of $0.1 \mathrm{ml}$ of blood was collected inserting a heparinized capillary tube $(1.15 \mathrm{~mm}$ diameter $)$ in the lateral canthus oriented toward the back of the head, gently pressured against the orbital bone just in front of the zygomatic arch until blood flows. After centrifugation (15 min at 2000 r.p.m.), plasma was stored at $-70^{\circ} \mathrm{C}$. PGE2 concentration analysis was performed using ELISA assay (ab133021-prostaglandin E2 Abcam ${ }^{\circledast}$ kit), and the resulting microplate was read at $405 \mathrm{~nm}$ using Skanlt software version 5.0.0.42 according to instructions from the manufacturer.

\subsection{Statistical Analysis}

Data were analyzed with GraphPad Software version 3.02 for Windows (GraphPad Software, San Diego, CA, USA) using one-way ANOVA followed by Tukey. Normality was tested using D'Angostino \& Pearson test. To analyze PGE2 plasma concentration, a Shapiro Wilk normality test was used and a two-way ANOVA. Differences between means with a $\mathrm{p}$ value $<0.05$ were considered significant at the $95 \%$ confidence level.

\section{Results}

\subsection{Carrageenan Administration Decreased Saccharine Consumption}

Rats that received carrageenan in the right hind-paw after deafferentation had a significant decrease in average saccharine consumption over the 7-day experiment $(81.9 \%$ vs $39.7 \%, \mathrm{p}<0.05)$ being a $52 \%$ change (Figure 2$)$. The decrease in saccharine consumption was apparent and significant $24 \mathrm{hrs}$ after carrageenan administration and remained significant until day 6 . On day 7 , there was still a lower consumption; however, it was not significant and seemed to be returning to pre-carrageenan levels (Figure 3 ).

We measured basal paw volume and edema; carrageenan caused a significant increase in paw volume compared to that of control from day 1 to 5 (Figure 4). We found an inverse correlation between edema and percentage of saccharine consumption $(\mathrm{r}=-0.5, \mathrm{p}<0.05)$ suggesting that degree of inflammation correlates with decrease in saccharine consumption (Figure 5).

\subsection{Carrageenan Administration after Deafferentation Had No Change in Thermal or Mechanical Withdrawal Latencies}

Animals that undergo deafferentation surgery (DS) significantly increased withdrawal latency in the plantar test model $(6.6$ vs $9.1 \mathrm{~s}, \mathrm{p}<0.05)$ while sham controls didn't (6.8 vs 5.9 s, p > 0.05). We found no significant differences in the Von Frey filament test (DS 33.3 vs $33.6 \mathrm{~g}, \mathrm{p}>0.05$ and sham 35.1 vs $32.3 \mathrm{~g}, \mathrm{p}>0.05$ ); this shows that deafferentation diminishes thermal nociception while mechanical nociception seems to be unaffected (Figure 6). 


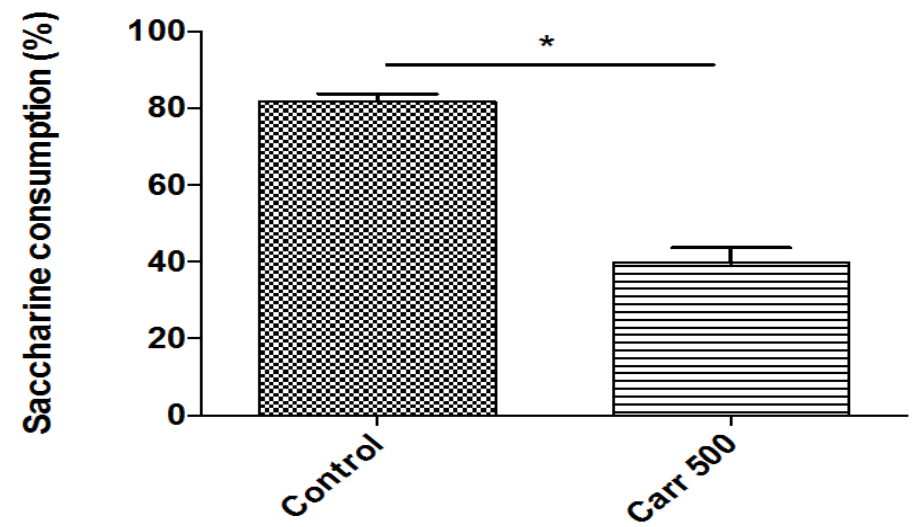

Figure 2. Average 7-day saccharine consumption after vehicle or carrageenan administration. Data represent the average percentage of daily consumption during 7 days for 8 10 rats. Statistical significance was determined using one way ANOVA and Tukey, ${ }^{*} \mathrm{p}<$ 0.05 . The administration of carrageenan significantly decreased average saccharine consumption with a $52 \%$ change.

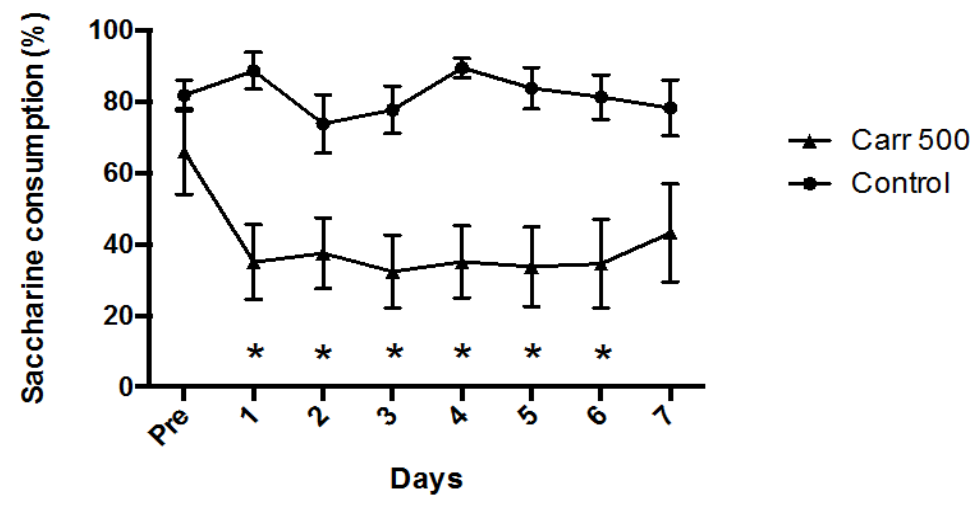

Figure 3. Daily saccharine consumption after vehicle or carrageenan administration. Data represent the daily consumption during 7 days for $8-10$ rats. Statistical significance was determined using two way ANOVA and Sidak, F $6.25{ }^{*} \mathrm{p}<0.05$. The administration of carrageenan caused a significant decrease in saccharine consumption from day 1 to day 6 , on day 7 the lower consume persisted but it was not significant, and consumption seemed to be recovering.

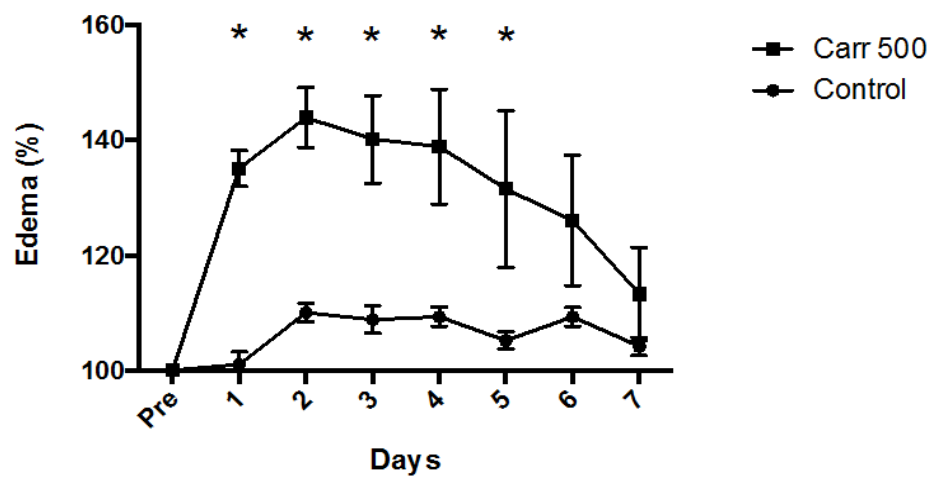

Figure 4. Daily paw edema after vehicle or $1 \%$ carrageenan administration. Data represent paw edema for 7 days for 8 - 10 rats. Statistical significance was determined using one way ANOVA and Tukey, ${ }^{*} \mathrm{p}<0.05$. The administration of carrageenan caused significant paw edema compared to vehicle from day 1 to day 5, decreasing on days 6 and 7 where the difference was not significative. 


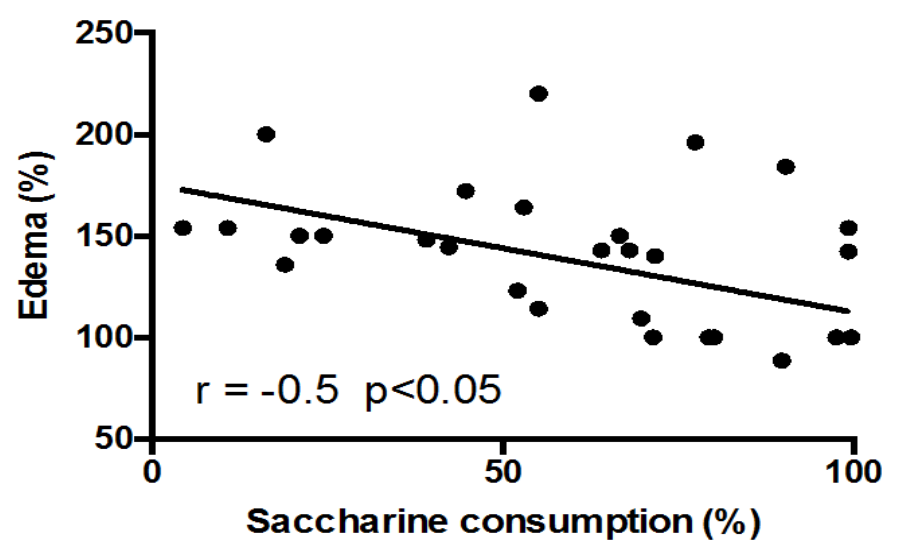

Figure 5. Correlation between paw edema and saccharine consumption. Each point represents the correlation between paw volume and \% saccharine consumption for control and carrageenan groups. Statistical significance was determined using Pearson test, ${ }^{\star} \mathrm{p}<$ 0.05. Paw edema and inflammation inversely correlates with saccharine consumption.

A

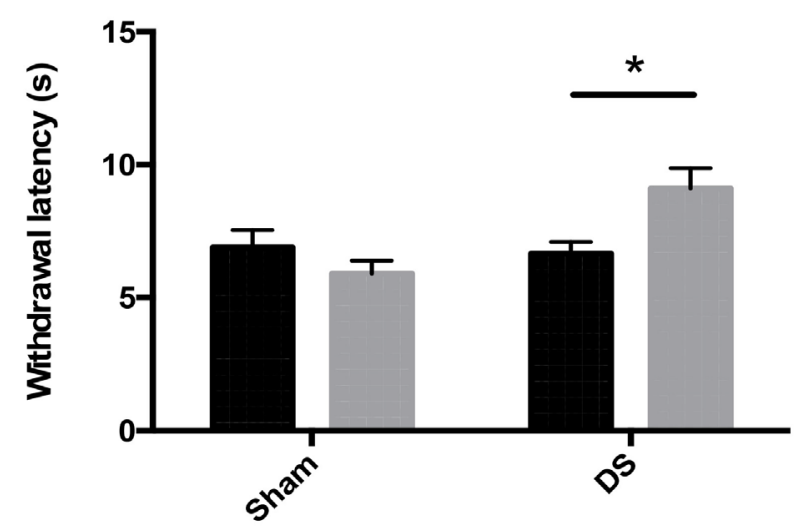

B

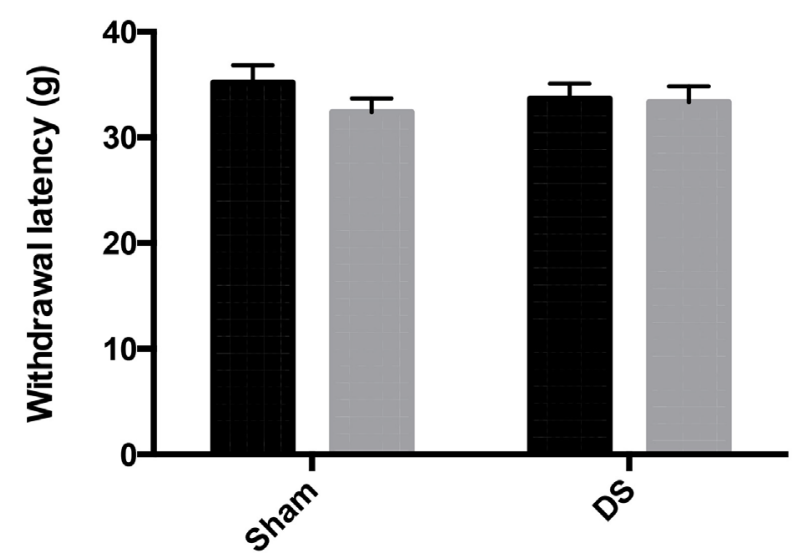

Figure 6. Withdrawal latency in sham and deafferentation surgery (DS) rats. A: Data represent average withdrawal latency for thermal nociception (plantar test) for 8 - 10 rats. B: Data represent average withdrawal latency for mechanical nociception (Von Frey) for 8 - 10 rats. Statistical significance was determined using one way ANOVA and Tukey, ${ }^{\star} \mathrm{p}<$ 0.05 . The deafferentation surgery significantly increased withdrawal latency for thermal nociception while mechanical nociception remained unchanged. 
The administration of carrageenan in the right hind paw after the deafferentation surgery did not change withdrawal latency to thermal nociception compared to that of rats that received saline ( $8.4 \mathrm{vs} 8.3 \mathrm{~s}, F=10.9, \mathrm{p}>0.05$ ). The carrageenan group had a significantly longer withdrawal latency compared to that of the sham group throughout the measurements ( 8.3 vs $6 \mathrm{~s}, F=10.9$, p $<0.005$ ). We did not find significant differences between groups in the withdrawal latency to mechanical nociception (Figure 7).

\subsection{Carrageenan Administration Did Not Alter PGE2 Concentrations}

Serum PGE2 concentrations were measure after 1,2,3 and 4 days post administration of carrageenan; statistical significance was determined using two way ANOVA and Tukey. Carrageenan administration didn't increase PGE2 concentration at any time compared with saline group (Figure 8).

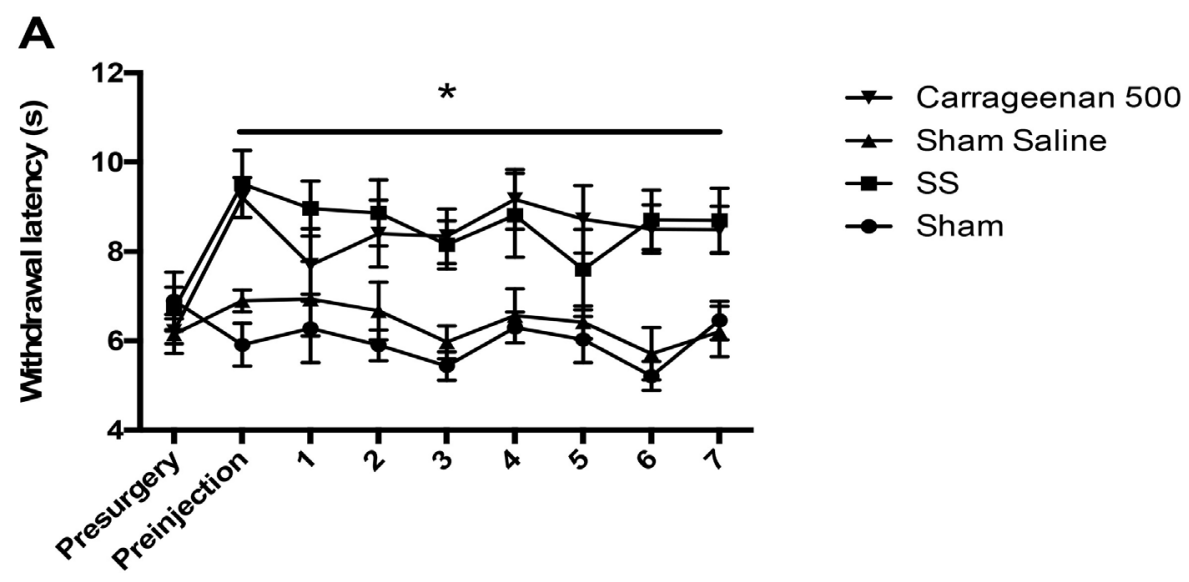

B

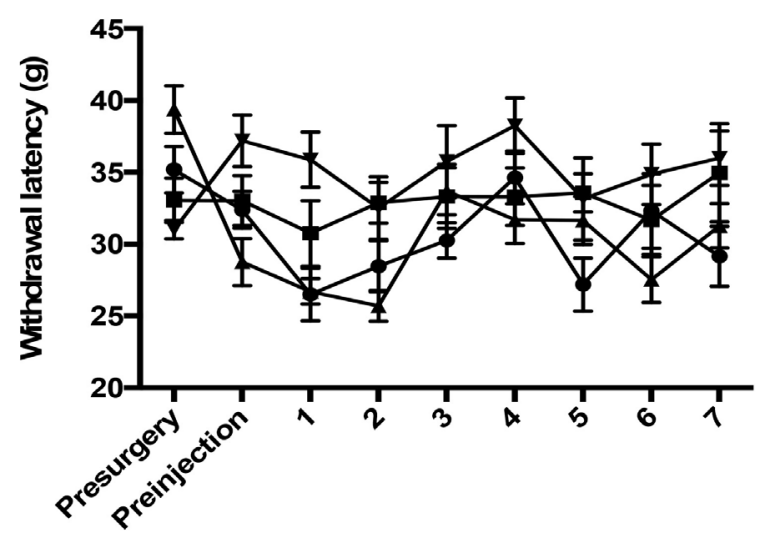

$\rightarrow$ Carrageenan 500

$\rightarrow$ Sham Saline

$\rightarrow$ SS

$\rightarrow$ Sham

Figure 7. Withdrawal latency in 7 day follow up in sham and deafferentation surgery (DS) rats. A: Data represent average withdrawal latency for thermal nociception (plantar test) for $8-10$ rats. B: Data represent average withdrawal latency for mechanical nociception (Von Frey) for 8 - 10 rats. Statistical significance was determined using two way ANOVA and Sidak, ${ }^{\star} \mathrm{p}<0.05$. Carrageenan administration didn't change thermal withdrawal compared to rats who received saline after deafferentation, both groups had significantly higher withdrawal latencies compared to rats without deafferentation; there were no differences in withdrawal latency in mechanical nociception. 


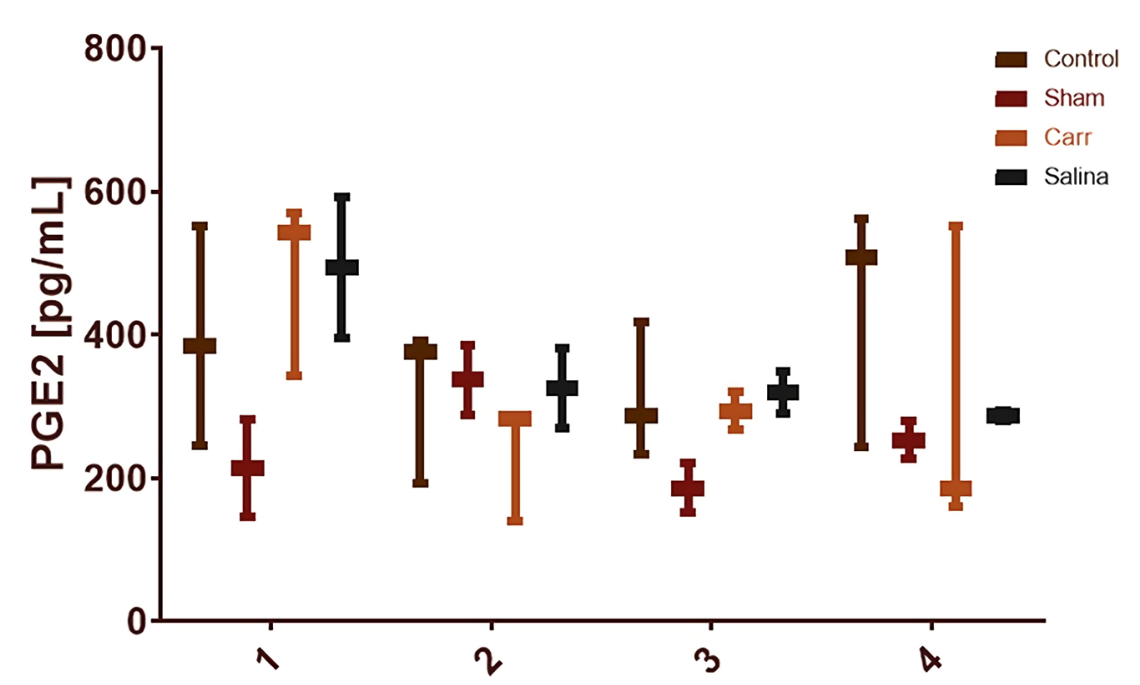

Figure 8. Prostaglandin concentration in control, sham, carrageenan and saline groups. Data represent average concentration of PGE2 in serum 1, 2, 3 and 4 days post administration.

\section{Discussion}

In the current research paper we show that carrageenan administration in the right hind paw after deafferentation surgery leads to anhedonia, which is evident by a decrease in saccharine consumption in a two-bottle choice test; our team had previously shown that inflammatory models for carrageenan administration prevent the development of morphine and nicotine conditioned place-preference, these findings suggest that carrageenan mediated inflammation is a reliable model of anhedonia that could lead to a better understanding of the immune-neural axis in motivation.

There is a mounting body of evidence showing that inflammation can lead to depression and anhedonia in animal models; rats that received an intraperitoneal injection of Calmette-Guerin bacillus (CGB) $1 \times 10^{7} \mathrm{CFU}$ presented depression in a forced swim model. This depressive conduct could be blocked with the administration of anti-IFN drugs showing a relevant role of intracerebral cytokines.

However, using bacteria as a neuro-immune model has a number of difficulties. CGB takes long to grow and requires a very controlled environment and it's antigenic structure is highly complex, leading to a high variability in immune response; whereas carrageenan is a polysaccharide easily obtainable and with a predictable immune response.

As aforementioned, carrageenan administration has previously shown to prevent conditioned place-preference responses to a number of substances including morphine, nicotine, cocaine and methamphetamine. However, with conditioned responses, a number of neural circuits participate including memory. Thus, to dissect the effect inflammation has over hedonic responses, we used the two bottle choice model, which is independent of memory.

We found that carrageenan administration in the right hind paw after deaffe- 
rentation surgery lead to a decrease of over half the rats' saccharine consumption; this decrease was proportional to the edema volume on the paw, showing a strong correlation between these two variables. In addition to this, while the inflammation decreased towards the latter days of the experiment, saccharine consumption went back up until no significant differences were found with regard to that of the control group, suggesting that this anhedonia model is reversible and allows repeated evaluations. Another confounding factor with previous inflammation-induced anhedonia models is pain since most of the inflammatory models such as formalin, which can lead to pain. Hence, we tested the administration after deafferentation surgery to validate that deafferentation is blocking the pain signal in our animal models. We also tested thermal and mechanical nociception in rats with the surgery and with the carrageenan administration.

We found that cutting the sciatic nerve leads to a significant decrease in thermal nociception evident by the fact that withdrawal latency in the plantar test model was significantly increased in the surgery and in the surgery + carrageenan animals; mechanical nociception, however, was not significantly affected. This could be due to the fact that the other paw nerves sense the movement in response to the Von Frey filament. Nevertheless, no rats with carrageenan administration after deafferentation surgery had a shorter withdrawal latency to heat or pressure compared to the sham groups, showing that the animals feel no pain.

In our previous work, ibuprofen managed to block carrageenan anhedonia in a conditioned place-preference model. This led us to believe that PGE2 might play a role in this effect, so we measured prostaglandin concentration in retroorbital blood. We did not find any significant differences, which could be due to the extraction technique used. Even though retroorbital blood samples are an easy and relatively innocuous way to obtain serial samples from the animal, there is tissular damage and an important breakage of blood vessels even when it is done right, this could account to the similar PGE2 concentrations in all of our groups. Another factor could be that PGE2 concentration peak before in our model and we could not detect it in the blood samples, this could suggest that other mediators are essential in the immune-brain communication causing this anhedonia.

We believe that this new model of immune anhedonia is a simple and reliable way to study how peripheral inflammation can affect mood and motivation. and further our understanding of the links between the immune system and the brain and potentially develop new treatments for depression and addiction.

\section{Acknowledgements}

This study was supported by UNAM-DGAPA-PAPIIT IN207918.

Authors want to thank Mrs. Josefina Bolado, Head of the Scientific Paper Translation Department, from División de Investigación at Facultad de Medicina, UNAM, for editing the English-language version of this manuscript.

\section{Conflicts of Interest}

The authors declare no conflicts of interest regarding the publication of this paper. 


\section{References}

[1] Otte, C., Gold, S.M., Penninx, B.W., Etkin, A., Fava, M., Mohr, D.C. and Schatzberg, A.F. (2016) Major Depressive Disorder. Nature Reviews Disease Primers, 15, 16065. https://doi.org/10.1038/nrdp.2016.65

[2] Hasin, D.S., Sarvet, A.L., Meyers, J.L., Saha, T.D., Ruan, W.J., Stohl, M. and Grant, B.F. (2018) Epidemiology of Adult DSM-5 Major Depressive Disorder and Its Specifiers in the US. JAMA Psychiatry, 75, 336-346.

https://doi.org/10.1001/jamapsychiatry.2017.4602

[3] Vrieze, E. (2013) Reduced Reward Learning Predicts Outcome in Major Depressive Disorder. Biology Psychiatry, 73, 639-645. https://doi.org/10.1016/j.biopsych.2012.10.014

[4] McMakin, D.L., Olino, T.M., Porta, G., Dietz, L.J., Asarnow, J.R., Ryan, N.D., Birmaher, B., Shamseddeen, W., Mayes, T., Kennard, B., Spirito, A., Keller, M., Lynch, F.L., Dickerson, J.F. and Brent, D.A. (2012) Anhedonia Predicts Poorer Recovery among Youth with Selective Serotonin Reuptake Inhibitor Treatment-Resistant Depression. Journal American Academy Children Adolescent Psychiatry, 51, 404-411. https://doi.org/10.1016/j.jaac.2012.01.011

[5] Keedwell, P.A., Andrew, C., Williams, S.C., Brammer, M.J. and Phillips, M.L. (2005) The Neural Correlates of Anhedonia in Major Depressive Disorder. Biology Psychiatry, 58, 843-853. https://doi.org/10.1016/j.biopsych.2005.05.019

[6] Keller, J., Gomez, R., Williams, G., Lambke, A., Lazzeroni, L., Murphy Jr., G.M. and Schatzberg, A.F. (2017) HPA Axis in Major Depression: Cortisol, Clinical Symptomathology and Genetic Variation Predict Cognition. Molecular Psychiatry, 22, 527-536. https://doi.org/10.1038/mp.2016.120

[7] Dean, J. and Keshavan, M. (2017) The Neurobiology of Depression: An Integrated Review. Asian Journal of Psychiatry, 27, 101-111. https://doi.org/10.1016/j.ajp.2017.01.025

[8] Knowland, D. and Lim, B.K. (2018) Circuit-Based Frameworks of Depressive Behaviors: The Role of Reward Circuitry and Beyond. Pharmacology Biochemistry and Behavior, 174, 42-52. https://doi.org/10.1016/j.pbb.2017.12.010

[9] Dantzer, R., Connor, J.C.O., Freund, G.G., Johnson, R.W. and Kelley, K.W. (2008) From Inflammation to Sickness and Depression: When the Immune System Subjugates the Brain. Nature Reviews Neuroscience, 9, 49-57. https://doi.org/10.1038/nrn2297

[10] Dooley, L.N., Kuhlman, K.R., Robles, T.F., Eisenberg, N.I., Craske, M.G. and Bower, J.E. (2018) The Role of Inflammation in Core Features of Depression: Insights from Paradigms Using Exogenously-Induced Inflammation. Journal of Neuroscience and Biobehavioral Reviews, 94, 219-237. https://doi.org/10.1016/j.neubiorev.2018.09.006

[11] De la Garza, R. (2005) Endotoxin- or Pro-Inflammatory Cytokine- Induced Sickness Behavior as an Animal Model of Depression: Focus on Anhedonia. Neuros cience and Biobehavioral Reviews, 29, 761-770.

https://doi.org/10.1016/j.neubiorev.2005.03.016

[12] Eisenberg, N.I., Berkman, E.T., Inagaki, T.K., Rameson, L.T., Mashal, N.M. and Irwin, M.R. (2010) Inflammation-Induced Anhedonia: Endotoxin Reduces Ventral Striatum Responses to Reward. Biological Psychiatry, 68, 748-754. https://doi.org/10.1016/j.biopsych.2010.06.010

[13] Bhattacharya, A., Lord, B., Grigoleit, J.S., He, Y., Fraser, I., Campbell, S.N., Taylor, N., Aluisio, L., O’Connor, J.C., Papp, M., Chrovian, C., Carruthers, N., Lovenberg, 
T.W. and Letavic, M.A. (2018) Neuropsychopharmacology of JNJ-55308942: Evaluation of a Clinical Candidate Targeting P2X7 Ion Channels in Animal Models of Neuroinflammation and Anhedonia. Neuropsychopharmacology, 43, 2586-2596. https://doi.org/10.1038/s41386-018-0141-6

[14] Yirmiya, R. (1996) Endotoxin Produces a Depressive-Like Episode in Rats. Brain Research, 711, 163-174. https://doi.org/10.1016/0006-8993(95)01415-2

[15] Burstein, O. and Doron, R. (2018) The Unpredictable Chronic Mild Stress Protocol for Inducing Anhedonia in Mice. Journal of Visualized Experiments, 140, e58184. https://doi.org/10.3791/58184

[16] Primeaux, S.D., Wilson, M.A., Wilson, S.P., Guth, A.N., Lelutiu, N.B. and Holmes P.V. (2003) Herpes Virus-Mediated Preproenkephalin Gene Transfer in the Ventral Striatum Mimics Behavioral Changes Produced by Olfactory Bulbectomy in Rats. Brain Research, 988, 43-45. https://doi.org/10.1016/S0006-8993(03)03337-7

[17] Willner, P., Daquila, P.S., Coventry, T. and Brain, P. (1995) Loss of Social Status: Preliminary Evaluation of a Novel Animal Model of Depression. Journal of Psychopharmacology, 9, 207-213. https://doi.org/10.1177/026988119500900302

[18] Suzuki, T., Kishimoto, Y. and Misawa, M. (1996) Formalin- and Carregeenan-Induced Inflammation Attenuates Place Preference Produced by Morphine, Methamphetamine and Cocaine. Life Science, 59, 1667-1674. https://doi.org/10.1016/0024-3205(96)00498-5

[19] Zimmermann, M. (1983) Ethical Guidelines for Investigations of Experimental Pain in Conscious Animals. Pain, 16, 109-110. https://doi.org/10.1016/0304-3959(83)90201-4

[20] Colleoni, M. and Sacerdote, P. (2010) Murine Models of Human Neuropathic Pain. Biochimica et Biophysica Acta, 1802, 924-933. https://doi.org/10.1016/j.bbadis.2009.10.012

[21] Amniattalab, A. and Mohammadi, R. (2017) Functional, Histopathological and Immunohistochemical Assessments of Cyclosporine A on Sciatic Nerve Regeneration Using Allografts: A Rat Sciatic Nerve Model. Bulletin of Emergency and Trauma, 5, 152-159.

[22] Ohtani, N. and Masaki, E. (2016) D2-Like Receptors in the Descending Dopaminergic Pathway Are Not Involved in the Decreased Postoperative Nociceptive Threshold Induced by Plantar Incision in Adult Rats. Journal of Pain Research, 9, 865-869. https://doi.org/10.2147/JPR.S120470

[23] Yamamoto, M., Motomura, E., Yanagisawa, R., Hoang, V.A.T., Mogi, M., Mori, T., Nakamura, M., Takeya, M. and Eto, K. (2019) Evaluation of Neurobehavioral Impairment in Methylmercury-Treated KK-Ay Mice by Dynamic Weight-Bearing Test. Journal of Applied Toxicology, 39, 221-230. https://doi.org/10.1002/jat.3710 